\title{
L'Acidosi Tubulare di tipo IV: una nefropatia emergente
}

\author{
Marco Quaglia, Guido Merlotti, Cristina Izzo, Piero Stratta
}

Dipartimento di Medicina Traslazionale, Unità di Nefrologia e Trapianto, A.O.U. "Maggiore della Carità", di Novara, Università “A. Avogadro”, Novara

TYPE IV RENAL TUBULAR ACIDOSIS: AN EMERGING TYPE OF NEPHROPATHY

Abstract. Type IV renal tubular acidosis (RTA IV) is a form of hyperchloremic RTA caused by an absolute or functional hypoaldosteronism that determines a distal acidification defect by inhibiting ammoniagenesis and $\mathrm{H}^{+}$excretion by the collecting duct. Etiology is often multifactorial and includes disorders causing a reduction in aldosterone levels or in the sensitivity of the collecting duct to the hormone. Several drugs block the renin-angiotensin system (RAS) at different steps, thus increasing the risk of developing RTA IV especially when used in association in elderly patients with diabetes, congestive heart failure, chronic renal failure, or in renal transplant recipients.

Diagnosis is based on the presence of hyperkaliemia disproportionate to the degree of renal function, associated with a metabolic acidosis with normal serum anion gap and a positive urinary anion gap; urinary pH can be lower than 5.5. Some laboratory features allow differential diagnosis with distal type I RTA. The clinical impact of RTA IV is becoming progressively heavier due to the widespread use of RAS-blocking drugs in elderly patients already at risk for this complication because of their comorbidities; this situation is inducing a critical revision of the indications to association therapies with these agents. The onset of hyperkalemia in this setting poses a therapeutic dilemma, as the patients at highest risk for RTA IV are also those who can derive the strongest cardiovascular benefits from RAS pharmacological blockade. It is therefore necessary to find a balance between risks and benefits in each patient, adopting preventive measures against RTA IV.

Key words: Renal tubular acidosis, Hyperkaliemia, Renin-angiotensin system, Aldosterone, Diabetes, Chronic renal failure

Conflict of interest: None.

Financial support: None.

Accettato: 15 Giugno 2014

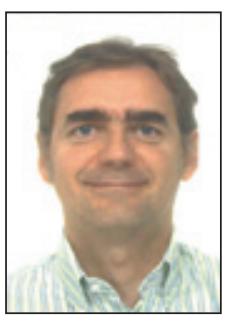

Marco Quaglia

\section{Introduzione}

L'acidosi tubulare (Renal Tubular Acidosis, RTA) è una forma di acidosi metabolica ipercloremica (con anion gap sierico normale) causata da un deficit nel riassorbimento di $\mathrm{HCO}_{3}^{-}$da parte del tubulo prossimale (RTA prossimale o tipo II) o nell'escrezione netta di $\mathrm{H}^{+}$da parte del tubulo distale (RTA distale), pur in presenza di un'acidosi (1-5). Benché alcune classificazioni tendano a considerarla un'unica entità (1), la RTA distale viene tradizionalmente distinta in (6):

- RTA tipo I (forma classica o ipokaliemica), causata da un difetto nella secrezione di idrogenioni che determina un'incapacità ad acidificare l'urina in modo massimale in condizioni di acidosi $(\mathrm{pH}$ urinario $>5.3$ );

- RTA tipo I (variante iperkaliemica), causata da un difetto nel riassorbimento distale di $\mathrm{Na}^{+}(\mathrm{pH}$ urinario $>5.3)$ e, da alcuni Autori (7), considerata parte della RTA di tipo IV;
- RTA tipo IV (iperkaliemica), causata principalmente da un deficit nell'ammoniogenesi secondario a ipoaldosteronismo e a iperkaliemia ( $\mathrm{pH}$ urinario di solito $<5.3$ ).

L'oggetto di questa review è la forma acquisita della RTA di tipo IV, che è aumentata considerevolmente nelle ultime decadi a causa del crescente impiego di farmaci che bloccano il sistema renina-angiotensina-aldosterone (RAS) a vari livelli in tipologie di pazienti già di per sé a rischio per questa complicanza (età avanzata, scompenso cardiaco, diabete mellito di tipo 2, insufficienza renale cronica) (4).

\section{Fisiopatologia}

Il meccanismo fisiopatologico fondamentale della RTA di tipo IV è costituito un deficit di acidificazione distale. Questo è causato da una riduzione dell'ammoniogenesi secondaria a un ipoaldosteronismo (assoluto o funzionale) e all'iperkaliemia. L'aldosterone condiziona il processo dell'ammoniogenesi e l'escrezione di $\mathrm{K}^{+}$attraverso varie azioni, che si esplicano sia 


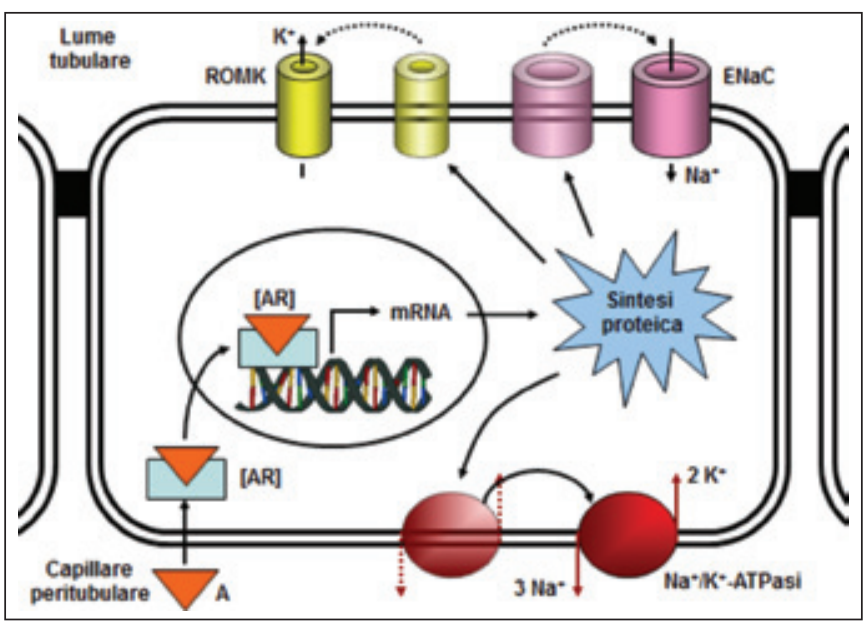

Fig 1: Azioni dell'aldosterone (A) sulla cellula principale del dotto collettore (schema semplificato).

a livello del dotto collettore che del tubulo contorto prossimale $(5,6)$.

1) A livello delle cellule principali del dotto collettore, l'aldosterone, dopo essersi legato al suo recettore, condiziona l'espressione e l'attività di alcune proteine cruciali per l'escrezione di $\mathrm{K}^{+}$e di $\mathrm{H}^{+}$(Fig. 1):

a) il canale epiteliale per il $\mathrm{Na}^{+}(\mathrm{ENaC})$, sul versante luminale della cellula tubulare, che ne consente l'ingresso passivo creando un potenziale elettrogenico negativo nel lume tubulare, essenziale sia per la secrezione passiva di $\mathrm{K}^{+}$(cfr. punto b) che per quella attiva di $\mathrm{H}^{+}$da parte delle cellule intercalate (cfr. punto 2); una ridotta attività intrinseca dell'ENaC oppure una ridotta disponibilità di $\mathrm{Na}^{+}$(per ridotto apporto e/o aumentato riassorbimento prossimale) inibiscono, quindi, indirettamente tutti questi processi ("voltage-dependent" RTA);

b) il canale epiteliale per il $\mathrm{K}^{+}$(ROMK), sempre sul versante luminale della cellula principale, che ne consente l'escrezione passiva, facilitata dal potenziale elettrogenico luminale negativo (6);

c) la pompa $\mathrm{Na}^{+} / \mathrm{K}^{+}$ATPasi, sul versante baso-laterale della cellula tubulare, che determina attivamente l'ingresso di ioni $\mathrm{K}^{+}$nella cellula tubulare e l'espulsione di ioni $\mathrm{Na}^{+}$ nei capillari peritubulari, creando i gradienti trans-cellulari di $\mathrm{Na}^{+}$e $\mathrm{K}^{+}$che consentono ai 2 canali luminali di riassorbire $\mathrm{Na}^{+}$e di espellere $\mathrm{K}^{+}$nel lume passivamente. Gli effetti finali dei processi descritti in a), b) e c) sono quindi il riassorbimento di $\mathrm{Na}^{+}$(dal lume al capillare peritubulare) e l'escrezione di $\mathrm{K}^{+}$e $\mathrm{H}^{+}$(in senso inverso). Un'alterazione a questo livello si manifesta clinicamente con iperkaliemia e acidosi tubulare (8).

2) A livello delle cellule intercalate di tipo $\alpha$ del dotto collettore, l'aldosterone determina l'espressione di diverse ATPasi $\left(\mathrm{H}^{+}\right.$ATPasi, $\mathrm{H}^{+} / \mathrm{K}^{+}$ATPasi) che secernono attivamente $\mathrm{H}^{+}$nel lume tubulare. Anche questo effetto è favorito dal potenziale elettrogenico negativo trans-epiteliale creato dall'ENaC a livello delle cellule principali (5).
3) A livello del tubulo prossimale, l'aldosterone determina la sintesi e la secrezione di $\mathrm{NH}_{3}$ che, agendo come buffer di $\mathrm{H}^{+}$nel lume tubulare, si trasforma in ione ammonio $\left(\mathrm{NH}_{4}^{+}\right)$. Questo viene riassorbito a livello dell'ansa di Henle (probabilmente sostituendo lo ione $\mathrm{K}^{+}$del trasportatore $\mathrm{Na}^{+} \mathrm{K}^{+}$ $2 \mathrm{Cl}^{-}$) e si dissocia parzialmente in $\mathrm{NH}_{3}$ e $\mathrm{H}^{+}$all'interno della cellula tubulare (a causa del più elevato $\mathrm{pH}$ intracellulare). L'ammoniaca diffonde quindi nell'interstizio della midollare, raggiungendo in questa sede elevate concentrazioni, e retro-diffonde in una seconda fase nelle cellule del dotto collettore e nel lume tubulare, seguendo il gradiente di $\mathrm{pH}$ (più basso nel lume del dotto collettore). In questa sede, $\mathrm{NH}_{3}$ tampona gli $\mathrm{H}^{+}$secreti dalle cellule intercalate di tipo $\alpha$ nel lume tubulare, ricostituendo $\mathrm{NH}_{4}^{+}$, che viene quindi "intrappolato" nel lume (non essendo liposolubile, a differenza di $\mathrm{NH}_{3}$ ) ed escreto. Una parte degli $\mathrm{NH}_{4}^{+}$riassorbiti nell'ansa di Henle sono invece espulsi come tali nell'interstizio, riassorbiti dalle cellule del dotto collettore e secreti nel lume tubulare, contribuendo direttamente all'eliminazione di valenze acide grazie all'idrogenione già compreso nella molecola.

L'iperkaliemia inibisce sia la sintesi di $\mathrm{NH}_{3}$ a livello del tubulo prossimale, sia i processi di riassorbimento e secrezione $\mathrm{NH}_{4}^{+}$(rispettivamente a livello dell'ansa di Henle e del dotto collettore) probabilmente per competizione tra $\mathrm{NH}_{4}^{+}$e $\mathrm{K}^{+}$per i medesimi trasportatori (6).

La marcata inibizione dell'ammoniogenesi da parte dall'iperkaliemia (9) sembra essere un meccanismo preponderante nel determinare la RTA di tipo IV e la differenzia da quella di tipo I (Tab. I), che è invece causata principalmente da un difetto nella secrezione di $\mathrm{H}^{+}$sia per una ridotta funzione della $\mathrm{H}^{+}$ATPasi delle cellule intercalate del dotto collettore ("secretion defect") che per un danno della membrana plasmatica della cellula tubulare, con retrodiffusione di $\mathrm{H}^{+}$secreti attivamente nel tubulo ("gradient defect"). La diversa eziopatogenesi condiziona le manifestazioni cliniche delle RTA: nel tipo I l'acidosi è più severa e il $\mathrm{pH}$ urinario è costantemente $>5.3$, mentre, nel tipo $\mathrm{IV}$, il difetto di acidificazione è in genere più lieve ed è causato da una ridotta disponibilità di $\mathrm{NH}_{3}$ endoluminale a livello del

TABELLA I - DIAGNOSI DIFFERENZIALE TRA LE ACIDOSI TUBULARI DISTALI

\begin{tabular}{|c|c|c|}
\hline & RTA tipo I & RTA tipo IV \\
\hline Eziopatogenesi & $\begin{array}{l}\text { Deficit secrezione } \\
\text { di } \mathrm{H}^{+}\end{array}$ & $\begin{array}{l}\text { Ipoaldosteronismo } \\
\text { e iperkaliemia }\end{array}$ \\
\hline $\mathrm{pH}$ urinario & $>5.5$ & Può essere $<5.5$ \\
\hline Bicarbonatemia & Anche $<10 \mathrm{mEq} / \mathrm{L}$ & $>15 \mathrm{mEq} / \mathrm{L}$ \\
\hline Potassiemia & Normale/Bassa & Elevata \\
\hline Funzione renale & Normale & Può essere ridotta \\
\hline $\mathrm{pCO}_{2}$ urinaria & $<40 \mathrm{mmHg}$ & $>70 \mathrm{mmHg}$ \\
\hline $\begin{array}{l}\text { Complicanze non } \\
\text { elettrolitiche }\end{array}$ & $\begin{array}{l}\text { Nefrocalcinosi, } \\
\text { nefrolitiasi }\end{array}$ & Nessuna \\
\hline
\end{tabular}


TABELLA II - RUOLO DEI FARMACI NELL'EZIOPATOGENESI DELLA RTA DI TIPO IV

\begin{tabular}{|c|c|c|}
\hline \multicolumn{3}{|c|}{ Ipoaldosteronismo assoluto } \\
\hline Farmaci & Meccanismo d'azione & Bibliografia \\
\hline $\begin{array}{l}\text { FANS, } \beta \text {-bloccanti, } \\
\text { inibitori diretti della } \\
\text { renina (DRI), inibitori } \\
\text { delle calcineurine }\end{array}$ & $\begin{array}{l}\text { Inibizione del rilascio } \\
\text { della renina }\end{array}$ & $(13,17,25-29)$ \\
\hline ACE-inibitori & $\begin{array}{l}\text { Inibizione della sintesi } \\
\text { di angiotensina } 2\end{array}$ & $(21-23,30,31)$ \\
\hline $\begin{array}{l}\text { Sartani, ketoconazolo, } \\
\text { eparina }\end{array}$ & $\begin{array}{l}\text { Inibizione della sintesi } \\
\text { surrenalica di aldosterone }\end{array}$ & $(13,17,31,32)$ \\
\hline \multicolumn{3}{|c|}{ Ipoaldosteronismo funzionale } \\
\hline Spironolattone, eplerenone & $\begin{array}{l}\text { Blocco del recettore per } \\
\text { l'aldosterone }\end{array}$ & $(33,34)$ \\
\hline $\begin{array}{l}\text { Amiloride, triamterene, } \\
\text { litio, pentamidina, } \\
\text { trimetoprim }\end{array}$ & $\begin{array}{l}\text { Blocco dell'ENaC } \\
\text { (cellule principali) }\end{array}$ & $(35-37)$ \\
\hline $\begin{array}{l}\text { Amiloride, litio, inibitori } \\
\text { delle calcineurine, } \\
\text { digitale }\end{array}$ & $\begin{array}{l}\text { Blocco della } \mathrm{Na}^{+} / \mathrm{K}^{+} \\
\text {ATPasi (cellule princi- } \\
\text { pali) }\end{array}$ & $(13,25-27,35-37)$ \\
\hline Amiloride, litio & $\begin{array}{l}\text { Blocco della } \mathrm{H}^{+} / \mathrm{K}^{+} \mathrm{ATPa}- \\
\text { si (cellule intercalate) }\end{array}$ & $(35-37)$ \\
\hline
\end{tabular}

dotto collettore, per cui i $\mathrm{H}^{+}$secreti nel lume non vengono tamponati. Pertanto, pur essendo l'escrezione acida netta complessivamente ridotta, il $\mathrm{pH}$ urinario può risultare $<5.3$ grazie alla presenza di idrogenioni liberi ("buffer defect"). Questo pattern riflette un'inibizione dell'ammoniogenesi tipica delle forme di RTA di tipo IV secondarie a ipoaldosteronismo assoluto, mentre il deficit nella secrezione di $\mathrm{H}^{+}$ assumerebbe maggiore importanza nelle forme di RTA di tipo IV secondarie a "resistenza" all'aldosterone a causa di un danno anatomico tubulo-interstiziale (ipoaldosteronismo "funzionale"); queste ultime sono caratterizzate quindi da un $\mathrm{pH}>5.3$, come si osserva nella RTA di tipo I (Tab. I).

La funzione dell'aldosterone è quindi cruciale per l'escrezione del $\mathrm{K}^{+}$(3-6). Questo ione, liberamente filtrato dal glomerulo, viene riassorbito per circa il $90 \%$ a livello del tubulo contorto prossimale e secreto in una misura pari a circa il $10 \%$ a livello del dotto collettore (l'escrezione frazionale del $\mathrm{K}^{+}$in presenza di un GFR normale è intorno al $10 \%$ ). $\grave{E}$ a questo livello che l'aldosterone realizza principalmente la regolazione dell'escrezione renale del $\mathrm{K}^{+}$, attraverso $\mathrm{i}$ meccanismi molecolari descritti. L'escrezione frazionale del $\mathrm{K}^{+}$costituisce un fondamentale meccanismo compensatorio, aumentando in modo inversamente proporzionale al livello di GFR e raggiungendo valori pari a circa il $40 \%$ quando il GFR si riduce al di sotto di $20 \mathrm{~mL} / \mathrm{min}$. Nell'insufficienza renale cronica avanzata, l'omeostasi del $\mathrm{K}^{+}$dipende in modo critico da questa funzione tubulare, che sopperisce al deficit di filtrazione glomerulare. L'inibizione farmacologica dell'aldosterone in questo contesto espone a un aumentato rischio di iperkaliemia, tanto maggiore quanto più il GFR è contratto (10).

\section{Eziologia}

Dal punto di vista eziologico è utile distinguere due fondamentali categorie di cause che possono determinare una RTA di tipo IV $(3,4,11)$ (Tab. II):

a) cause che determinano un'inibizione del RAS a livello dell'apparato iuxtaglomerulare (ipoaldosteronismo iporeninemico) o della surrene (ipoaldosteronismo primitivo), caratterizzate da bassi livelli di aldosterone con livelli di renina ridotti (nel primo caso) o aumentati (nel secondo);

b) cause che determinano un'inibizione della risposta all'aldosterone a livello tubulare ("resistenza all'aldosterone"), configurando un ipoaldosteronismo "funzionale" pur in presenza di livelli circolanti elevati.

Analizzeremo le principali cause di RTA di tipo IV afferenti alla prima categoria, caratterizzate da un ipoaldosteronismo (a):

1) una ridotta secrezione di renina da parte dell'apparato iuxtaglomerulare causata da un'alterazione nell'attività simpatica è tipica della nefropatia diabetica, responsabile del $60 \%$ dei casi di ipoaldosteronismo iporeninemico (12). Alcune nefropatie che coinvolgono in modo particolare l'apparato iuxtaglomerulare possono determinare una RTA di tipo IV: tra queste, l'uropatia ostruttiva, la nefropatia da analgesici, la nefropatia lupica, la nefropatia in corso di HIV, l'amiloidosi renale e la nefropatia cronica da trapianto (11). Diversi farmaci possono inibire la produzione di renina da parte dell'apparato iuxtaglomerulare: inibitori diretti della renina (DRI), beta-bloccanti (inibizione della stimolazione simpatica sull'apparato iuxtaglomerulare) FANS (inibizione della prostaciclina), inibitori delle calcineurine o CNI (ciclosporina e tacrolimus) (13);

2) una ridotta secrezione di aldosterone da parte della surrene nonostante livelli normali/elevati di renina è causata da tutte le forme di iposurrenalismo (p. es., Addison) e da diversi farmaci: ACE-inibitori (inibizione della sintesi di angiotensina 2), sartani (blocco del recettore surrenalico per l'angiotensina 2), eparina e ketoconazolo (inibizione della sintesi surrenalica di aldosterone) $(11,13)$.

Le principali cause di RTA di tipo IV secondaria a una "resistenza" all'aldosterone da parte del tubulo renale (b) sono costituite dalla presenza di un danno tubulo-interstiziale che coinvolge il dotto collettore, come accade in molte forme di insufficienza renale cronica (14), oppure da cause farmacologiche: anti-aldosteronici (blocco del recettore per l'aldosterone), amiloride, triamterene, trimetoprim, pentamidina, litio, inibitori delle calcineurine, litio (blocco del canale $\mathrm{ENaC}$ sulle cellule principali del dotto collettore, con conseguente "voltage defect") (11) e digitale (blocco della $\mathrm{Na}^{+}$/ $\mathrm{K}^{+}$ATPasi).

L'eziologia della RTA di tipo IV è quindi spesso multifattoriale, in quanto diversi contesti favorenti possono coesistere 
nel medesimo paziente, come esemplificato dalle seguenti condizioni:

1) età avanzata

2) diabete mellito

3) scompenso cardiaco

4) insufficienza renale cronica

5) trapianto renale

6) farmaci

1) Età avanzata. Il rene senile è a rischio di RTA di tipo IV a causa di vari fattori: l'apparato iuxtaglomerulare è spesso meno attivo nella secrezione di renina a causa di un deficit nella stimolazione simpatica (12), mentre è frequente la presenza di un danno tubulo-interstiziale cronico che determina un ridotta risposta del dotto collettore all'aldosterone $(15,16)$. La presenza di atrofia tubulare e fibrosi interstiziale può essere significativa anche in presenza di una normale funzione renale e riflette alterazioni della rete capillare peritubulare (17). L'elevata frequenza di un'uropatia ostruttiva secondaria a ipertrofia prostatica nell'anziano contribuisce all'eziopatogenesi di forme tubulo-interstiziali croniche, determinando anche un ampio spettro di difetti specifici, tra i quali una ridotta funzione dell' $\mathrm{ENaC}$ e della $\mathrm{Na}^{+} / \mathrm{K}^{+}$ATPasi con conseguente "voltage-defect" $(18,19)$. Inoltre, l'ipodipsia dell' anziano può creare una condizione di ipovolemia con riduzione del carico di $\mathrm{Na}^{+}$a livello del dotto collettore e ridotta funzione dell' $\mathrm{ENaC}$, aggravando il "voltage-defect" (11).

2) Diabete mellito. Il diabete mellito rappresenta un fattore favorente la RTA di tipo IV sia attraverso un'inibizione funzionale del RAS a causa della cronica espansione volemica sia a causa della nefropatia diabetica che coinvolge sia l'apparato iuxtaglomerulare (ridotta secrezione di renina) che il settore tubulo interstiziale (resistenza all' aldosterone) $(11,12,14)$. È inoltre spesso presente un'atrofia della surrene che la rende ipo-responsiva all'angiotensina 2 (ipoaldosteronismo primario) (20). Nelle fasi di scompenso glicemico con poliuria, anche l'ipovolemia può aggravare la RTA di tipo IV. Infine l'iperkaliemia può essere amplificata dalla condizione di insulino-resistenza, che ostacola lo shift intracellulare del $\mathrm{K}^{+}(11)$.

3) Scompenso cardiaco congestizio. Questa condizione espone al rischio di RTA di tipo IV soprattutto per cause emodinamiche e farmacologiche. La terapia dello scompenso cardiaco è basata sull'inibizione del RAS con ACE-inibitori, sartani e antialdosteronici, impiegati singolarmente o in associazione $(21,22)$. In condizioni di compenso emodinamico, l'ipoaldosteronismo funzionale indotto da questi farmaci è bilanciato dai loro effetti positivi sulla funzione sistolica e quindi sulla volemia efficace. La migliore perfusione renale che ne consegue attenua a sua volta l'attivazione del RAS che è tipica dello scompenso cardiaco congestizio (iperaldosteronismo secondario). L'effetto combinato di una migliore perfusione renale e della riduzione dei livelli di angiotensina 2 determina un'attenuazione del riassorbimento di acqua e $\mathrm{Na}^{+}$a livello del tubulo contorto prossimale e una maggiore disponibilità distale di $\mathrm{Na}^{+}$. A livello del dotto collettore, l'ENaC, pur essendo scarsamente espresso sulle cellule principali a causa dell'ipoaldosteronismo "farmacologico", è comunque in grado di creare un voltaggio negativo trans-epiteliale grazie al riassorbimento passivo di un'adeguata quantità di $\mathrm{Na}^{+}$, creando così le condizioni per l'escrezione di $\mathrm{K}^{+}$ e la secrezione di $\mathrm{H}^{+}$. Questa condizione di equilibrio è fondata sul mantenimento di un buon compenso emodinamico e di un'adeguata perfusione renale. Qualora il compenso emodinamico e la perfusione renale si deteriorino (a causa di un peggioramento della funzione sistolica oppure di un'ipovolemia) e la disponibilità di $\mathrm{Na}^{+}$al nefrone distale si riduca al di sotto di una soglia critica, identificata da una sodiuria $<10 \mathrm{mEq} / \mathrm{L}$, il rischio di RTA di tipo IV aumenta, in quanto il blocco farmacologico del RAS non consente margini di adattamento alla riduzione del carico di $\mathrm{Na}^{+}$disponibile a livello del dotto collettore (11). In questa sede un secondo fattore limitante l'escrezione di $\mathrm{K}^{+}$ è la riduzione della flusso della pre-urina, che rallenta il "wash-out" degli ioni $\mathrm{K}^{+}$dal brush-border in prossimità dei canali del $\mathrm{K}^{+}(5)$. Quando il compenso emodinamico si deteriora, spesso i farmaci bloccanti il RAS causano un peggioramento della funzione renale, con un ulteriore aggravamento dell'iperkaliemia, in quanto la riduzione della pressione intraglomerulare non è più bilanciata da un'adeguata perfusione glomerulare (23).

4) Insufficienza renale cronica. Nell'insufficienza renale cronica, soprattutto quando il GFR scende al di sotto di $30 \mathrm{~mL} / \mathrm{min}$, l'escrezione frazionale di $\mathrm{K}^{+}$aumenta per garantire un'adeguata eliminazione dello ione nonostante la riduzione nei nefroni residui $(6,10)$. Questo meccanismo adattativo dipende dalle azioni dell'aldosterone sul tubulo renale (cfr. Fisiopatologia), per cui un blocco del RAS espone il paziente al rischio di sviluppare RTA di tipo IV (14). Questa complicanza è più frequente in alcune nefropatie che coinvolgono il settore tubulo-interstiziale, tra le quali la nefropatia da analgesici, la nefropatia ostruttiva, la nefrite lupica e la nefrite in corso di HIV (11).

5) Trapianto renale. La RTA di tipo IV è presente in un'elevata percentuale di pazienti portatori di trapianto renale (fino al 40\%), spesso in forma lieve (24). Nell'immediato post-trapianto può essere espressione di una necrosi tubulare acuta, mentre, in fase tardiva, la RTA è stata associata al rigetto acuto, all'età del donatore, alla presenza di una funzione renale subottimale e alla nefrotossicità da CNI (25). Questi farmaci da un lato esercitano un'inibizione diretta sulla secrezione di renina da parte dell'apparato iuxtaglomerulare e, dall'altro, determinano un danno tubulo-interstiziale cronico con conseguente ridotta risposta all'aldosterone. Inoltre la ciclosporina inibisce l'attività della peptidil-prolil cis-trans isomerasica (PPIasi) della ciclofillina, che è essenziale per la trasformazione delle cellule intercalate da tipo $\beta$ (deputate alla secrezione di $\mathrm{HCO}_{3}^{-}$) a tipo $\alpha$ (deputate alla secrezione di protoni) (26). Altri possibili fattori favorenti la RTA di tipo IV nel rene trapiantato sono costituiti dalla presenza di una componente ostruttiva e dalla terapia con steroide (inibizione della sintesi di $\mathrm{NH}_{4}^{+}$a livello del tubulo prossimale), cotrimossazolo o pentamidina (inibizione dell' $\mathrm{ENaC}$ a livello delle 
cellule principali del dotto collettore), ACE-inibitori e sar$\operatorname{tani}(27)$.

6) Farmaci. I farmaci bloccanti il RAS a qualunque livello, soprattutto quando impiegati in associazione in pazienti affetti da una delle precedenti condizioni, espongono a un aumentato rischio di RTA di tipo IV (Tab. II). I meccanismi di blocco farmacologico sono molteplici, dall'inibizione del rilascio della renina da parte dell'apparato iuxtaglomerulare (blocco "prossimale") (25-29) all'inibizione della sintesi surrenalica di aldosterone (30-32), fino al blocco del recettore dell'aldosterone $(33,34)$ e dei canali e delle $\mathrm{Na}^{+} / \mathrm{K}^{+}$ATPasi espresse sulle cellule tubulari del dotto collettore (blocco "distale") (35-37).

\section{Inquadramento diagnostico}

L'acidosi tubulare di tipo IV è un disordine caratterizzato da un'acidosi metabolica ipercloremica ad anion gap (AG) sierico normale e da un AG urinario (uAG) positivo (38). Le due formule relative sono rispettivamente:

$$
\begin{aligned}
& \text { AG sierico: }\left[\mathrm{Na}^{+}\right]-\left(\left[\mathrm{Cl}^{-}\right]+\left[\mathrm{HCO}_{3}^{-}\right]\right) \\
& \text {AG urinario }(\mathrm{uAG}):\left(\left[\mathrm{uNa}^{+}\right]+\left[\mathrm{uK}^{+}\right]\right)-\left[\mathrm{uCl}^{-}\right]
\end{aligned}
$$

La presenza di un AG sierico normale con ipercloremia consente la diagnosi differenziale tra tutte le forme di RTA e l'acidosi metabolica secondaria all'insufficienza renale cronica avanzata e con altre forme di acidosi metabolica a eziologia extra-renale come l'acidosi lattica e la chetoacidosi diabetica (tutte caratterizzate da un AG sierico aumentato).

La positività dell'uAG consente di distinguere le forme di RTA distale dalla RTA prossimale, caratterizzata invece da un uAG negativo. Infatti, l'uAG fornisce una stima dell'escrezione urinaria di $\mathrm{NH}_{4}^{+}$: nell'acidosi metabolica questa aumenta, determinando un'aumentata escrezione urinaria $\mathrm{di}^{-} \mathrm{Cl}^{-}$e quindi una negativizzazione dell'uAG che, in condizioni normali, è lievemente positivo (28). In presenza di un difetto nell'acidificazione distale (deficit di ammoniogenesi e/o di secrezione di $\mathrm{H}^{+}$), l'uAG rimane inadeguatamente positivo nonostante l'acidosi metabolica, mentre il difetto nel riassorbimento di $\mathrm{HCO}_{3}^{-}$che caratterizza la RTA prossimale ne consente la negativizzazione $(4,8,39)$.

Inquadrata la RTA come forma distale, è necessario eseguire una diagnosi differenziale tra RTA di tipo I e di tipo IV, in base ad alcune caratteristiche clinico-laboratoristiche (Tab. I): in particolare i livelli di kaliemia, sempre elevati nel tipo IV e spesso ridotti nel tipo I (almeno nella forma "classica"), e il $\mathrm{pH}$ urinario, che può essere inferiore a 5.5 nel tipo IV (soprattutto nelle forme "pure" di ipoaldosteronismo iporeninemico) mentre è sempre tipicamente superiore a questa soglia nel tipo I. Queste differenze riflettono la fisiopatologia dei due disordini: difetto isolato di secrezione di $\mathrm{H}^{+}$nella RTA di tipo I e prevalente difetto dell'ammoniogenesi sostenuto dall'iperkaliemia nella RTA di tipo IV (3-8).

La "variante iperkaliemica" della RTA di tipo I costituisce in effetti una forma intermedia tra le due acidosi tubulari distali. Questa riconosce come "primum movens" eziopatogenetico un difetto nel riassorbimento del $\mathrm{Na}^{+}$da parte dell' $\mathrm{ENaC}$ della cellula principale del dotto collettore (Fig. 1), che determina una ridotta elettronegatività endoluminale con conseguente ridotta escrezione urinaria di $\mathrm{K}^{+}$e iperkaliemia. È stata descritta nella nefropatia ostruttiva (nella quale è concomitante anche un deficit dell'attività della pompa $\mathrm{Na}^{+} / \mathrm{K}^{+}$ATPasi basolaterale della cellula principale a causa di un danno tubulointerstiziale, Fig. 1), nella nefrite lupica (nella quale l'attività $\mathrm{H}^{+}$ATPasica delle cellule intercalate è solitamente conservata) e nell'anemia falciforme (nella quale può coesistere un ipoaldosteronismo) oltre che nell'ipovolemia severa (a causa del ridotto carico di $\mathrm{Na}^{+}$endoluminale a livello del dotto collettore) $(3,5-8)$.

Al fine di confermare la diagnosi differenziale di queste diverse forme di RTA distale è infine possibile eseguire un test di acidificazione, che consiste nel somministrare un carico acido al fine di valutare la capacità del tubulo di ridurre il $\mathrm{pH}$ urinario in modo massimale $(\mathrm{pH}$ urinario $<5.5)$. Il carico acido veniva tradizionalmente somministrato come $\mathrm{NH}_{4} \mathrm{Cl}$ per via orale, mentre un test più recente e agevole (40) consiste nella somministrazione orale simultanea di furosemide $(40 \mathrm{mg})$, per aumentare la disponibilità di $\mathrm{Na}^{+}$a livello del dotto collettore, e di fludrocortisone $(1 \mathrm{mg})$, per stimolare con un effetto mineralcorticoide il riassorbimento di $\mathrm{Na}^{+}$da parte delle cellule principali e la secrezione di $\mathrm{H}^{+}$da parte delle cellule intercalate di tipo $\alpha$. Il $\mathrm{pH}$ urinario viene misurato prima della somministrazione dei farmaci e successivamente ogni ora per 6 ore. Si profilano a questo punto 3 possibilità:

1) se la kaliemia è normale o ridotta e il test di acidificazione documenta l'incapacità a ridurre il $\mathrm{pH}$ urinario al di sotto di 5.5 si conferma la diagnosi di RTA di tipo I (deficit di secrezione di $\mathrm{H}^{+}$);

2) se la kaliemia è aumentata e il test di acidificazione determina una riduzione del $\mathrm{pH}$ urinario $<5.5$, si conferma la diagnosi di RTA di tipo IV;

3) se la kaliemia è aumentata, ma il test di acidificazione documenta l'incapacità a ridurre il $\mathrm{pH}$ urinario al di sotto di 5.5 , il quadro è compatibile con la cosiddetta variante iperkaliemica della RTA di tipo I oppure con quelle forme di RTA di tipo IV causate da un danno anatomico tubulointerstiziale con resistenza all'aldosterone (piuttosto che da un ipoaldosteronismo assoluto), nelle quali il deficit di secrezione di $\mathrm{H}^{+}$è un meccanismo prevalente sull'inibizione dell'ammoniogenesi.

Da questa analisi si evince come né la kaliemia né il pH urinario siano sufficienti a distinguere in modo assoluto tra RTA di tipo I e RTA di tipo IV, in quanto entrambe sono caratterizzate da varianti (la forma "iperkaliemica" di RTA di tipo I e la forma secondaria a "resistenza all'aldosterone" per danno tubulare nella RTA di tipo IV) che si discostano dal pattern delle due forme classiche per entrambi questi parametri.

In genere, tuttavia, la diagnosi di RTA di tipo IV è facilitata da un contesto clinico suggestivo, che rende superflua l'esecuzione di test funzionali. Il dosaggio dei livelli di renina e aldosterone plasmatici non è essenziale perché i valori di aldosterone potrebbero essere normali in presenza di forme di RTA di tipo IV secondarie a "resistenza" tubulare all'ormone. 
Anche nella RTA di tipo I, il contesto clinico, soprattutto nelle forme complete, indirizza facilmente verso la diagnosi, essendo caratterizzato da quadri di nefrolitiasi (calcoli di fosfato di calcio o struvite) e nefrocalcinosi, favorite dalle alterazioni del quadro urinario: ipercalciuria, iperfosfaturia, ipocitraturia (secondaria all'ipokaliemia e all'acidosi metabolica) e $\mathrm{pH}$ persistentemente elevato $(3,5-8)$.

\section{Manifestazioni cliniche}

Il quadro clinico della RTA di tipo IV (Tab. I) è costituito da un'iperkaliemia sproporzionata rispetto alla funzione renale (che è normale o moderatamente contratta) e preminente rispetto a un'acidosi metabolica ipercloremica in genere moderata $\left(\mathrm{HCO}_{3}^{-}>15 \mathrm{mEq} / \mathrm{L}\right)$, con $\mathrm{AG}$ sierico normale. Il $\mathrm{pH}$ urinario può essere $<5.5$ (soprattutto nell'ipoaldosteronismo iporeninemico) e l'uAG è inadeguatamente positivo. Il quadro sintomatologico è in genere dominato dalle manifestazioni dell'iperkaliemia (debolezza muscolare, bradicardia), che può essere severa. Spesso il paziente è anziano e affetto da diabete mellito (circa $50 \%$ dei casi), la causa più frequente di ipoaldosteronismo iporeninemico. Più raramente l'ipoaldosteronismo è primitivo ed è causato da un'insufficienza surrenalica, espressione o di un'atrofia della zona glomerulosa secondaria a insulino-resistenza in corso di diabete oppure di altri processi patologici a carico della ghiandola, come, per esempio, nell'HIV (41).

Possono coesistere condizioni favorenti di tipo emodinamico, con riduzione della volemia efficace (scompenso cardiaco congestizio, ipovolemia). La funzione renale può essere normale, ma più spesso è presente una lieve insufficienza renale cronica secondaria a nefropatia diabetica oppure a una nefrite tubulo-interstiziale cronica. Nell'anziano è da ricercare una componente ostruttiva cronica, che può causare una ridotta escrezione di $\mathrm{H}^{+}$a causa di un difetto "voltage-dependent" $(15,17-19)$ ed è frequente un fattore favorente farmacologico (Tab. II).

\section{Impatto clinico e prevenzione della RTA di tipo IV}

La RTA di tipo IV è una tubulopatia emergente da un lato a causa dell'invecchiamento della popolazione e dell'elevata prevalenza delle condizioni a rischio per questa complicanza (diabete di tipo 2, scompenso cardiaco, insufficienza renale cronica) e, dall'altro, per la diffusione di farmaci bloccanti il RAS in questi medesimi pazienti, in genere con indicazioni cardio-vascolari $(21-23,29,30)$. Spesso coesistono due o più di queste condizioni: il $30-50 \%$ dei pazienti con scompenso cardiaco congestizio ha anche un'insufficienza renale cronica ed entrambe le comorbidità sono frequenti nel diabetico (21). Si tratta quindi di pazienti complessi, spesso trattati con una politerapia ipotensiva.

Una delle prime segnalazioni del rischio di indurre una RTA di tipo IV attraverso un'associazione di farmaci bloccanti il RAS è stata quella di Juurlink DN et al: un allarmante incremento di episodi di iperkaliemia fatale si è verificato dopo la diffusione dello studio RALES (Randomized Aldactone Evaluation
Study) (34), che aveva dimostrato una riduzione del 30\% della mortalità aggiungendo lo spironolattone a una terapia con ACE-inibitore nello scompenso cardiaco severo. All'origine di molti casi di iperkaliemia era l'impiego di questa associazione in pazienti già a rischio di RTA di tipo IV per le loro caratteristiche cliniche, con un dosaggio dell'antialdosteronico superiore a quello impiegato nello studio $(25 \mathrm{mg} /$ die $) \mathrm{e}$ un monitoraggio insufficiente dell'evoluzione della kaliemia dopo l'introduzione del farmaco, soprattutto in presenza di un peggioramento della funzione renale $(21,23)$. Questi elementi esemplificano i rischi di un impiego estensivo delle associazioni di farmaci bloccanti il RAS nella realtà clinica, in condizioni spesso differenti da quelle degli studi (42).

Anche l'associazione di ACE-inibitore e sartano, valutata nello studio ONTARGET (Ongoing Telmisartan Alone and in Combination with Ramipril Global Endpoint Trial) ha determinato una significativa incidenza di iperkaliemia e insufficienza renale acuta, complicanze che hanno contribuito a metterne in discussione il reale vantaggio rispetto alla terapia con i due farmaci impiegati singolarmente (22, 29, 30).

Infine, l'associazione tra inibitore diretto della renina (DRI) e sartano è stata controindicata nei pazienti diabetici e con insufficienza renale per l'elevato rischio di eventi avversi (tra i quali l'iperkaliemia), che hanno determinato l'interruzione dello studio ALTITUDE (Aliskiren trial in type 2 diabetes using cardio-renal endpoints) $(43,44)$.

Peraltro, un'iperkaliemia si sviluppa in circa il 10\% dei pazienti entro 1 anno dall'avvio di una monoterapia con solo ACE-inibitore $(11,13)$.

È da sottolineare come tutti questi studi si siano focalizzati sui due end-point clinicamente più rilevanti, l'insorgenza di iperkaliemia e il peggioramento della funzione renale, e non forniscano dati esaurienti sulle alterazioni del quadro acidobase dopo l'avvio dei farmaci bloccanti del RAS. Tuttavia questi farmaci sono considerati come fattori predisponenti alla RTA di tipo IV in quanto, attraverso la riduzione della sintesi o dell'azione dell'angiotensina 2, principale stimolo alla secrezione surrenalica di aldosterone, creano uno stato di ipoaldosteronismo che priva il paziente di un fondamentale meccanismo dell'omeostasi del potassio e dell'equilibrio acido-base (Tab. II).

Il blocco farmacologico del RAS predispone quindi all'insorgenza di RTA di tipo IV soprattutto in alcuni contesti clinici come il diabete, lo scompenso cardiaco e l'insufficienza renale cronica, nei quali l'insorgenza di iperkaliemia pone un dilemma terapeutico: proprio i pazienti a maggior rischio per RTA di tipo IV sono in effetti quelli che possono trarre il massimo beneficio cardio-vascolare dal blocco del RAS $(11,21)$. É, quindi, necessario trovare un punto di equilibrio nel rapporto rischio/beneficio nel singolo paziente, adottando misure di prevenzione della RTA di tipo IV che possano consentire un impiego sicuro di questi farmaci (11):

1) è necessario eseguire una corretta stima del filtrato glomerulare (eGFR) con l'impiego della formula di Cockcroft oppure del BIS-1. Quest'ultimo appare più attendibile della prima nel paziente di età superiore ai 70 anni con CKD allo stadio 1-3 (45); 
2) nel paziente anziano vasculopatico con ipertensione arteriosa importante occorre prendere in considerazione l'esecuzione di un Ecodoppler delle arterie renali per escludere una malattia renale ischemica $(15,23)$;

3) un'attenta anamnesi farmacologica è fondamentale per valutare le interazioni tra farmaci responsabili di RTA di tipo IV, inclusi i FANS, di frequente impiego nel paziente anziano;

4) nei pazienti con insufficienza renale cronica e associata acidosi metabolica è utile l'impiego del $\mathrm{NaHCO}_{3}$ per via orale (dose media: $1 \mathrm{~g} /$ die), con il duplice obiettivo di aumentare la disponibilità di $\mathrm{Na}^{+}$a livello del tubulo distale e di favorire lo shift intracellulare di $\mathrm{K}^{+}$. L'associazione di questa terapia con un diuretico consente di prevenire il sovraccarico idrovolemico e di potenziare ulteriormente il carico distale di $\mathrm{Na}^{+}$, promuovendone il riassorbimento da parte delle cellule principali del dotto collettore e creando quindi le premesse per l'escrezione di $\mathrm{K}^{+}$e $\mathrm{H}^{+}$. È consigliabile una restrizione dietetica di $\mathrm{K}^{+}$;

5) la riduzione dell'iperkaliemia di per sé (p. es., con terapia idratante e diuretica) può ridurre la severità dell'acidosi metabolica o persino risolverla completamente in assenza di insufficienza renale, poiché rimuove l'inibizione dell'iperkaliemia sull'ammionogenesi $(7,9)$;

6) la terapia con ACE-inibitore o sartano dovrebbe essere iniziata al dosaggio minimo (p. es., ramipril $1.25 \mathrm{mg}$ ), misurando la kaliemia dopo 1 settimana dall'avvio o dopo averne aumentato la posologia. Se la kaliemia si mantiene normale, la posologia del farmaco può essere aumentata progressivamente. Se la kaliemia aumenta e si colloca tra 5-5.5 mEq/L, è necessario ridurre la posologia del farmaco e sospendere altri farmaci bloccanti il RAS eventualmente associati. Se la kaliemia supera il valore di 5.5-6 $\mathrm{mEq} / \mathrm{L}$, è opportuno sospendere il farmaco o i farmaci completamente e re-introdurli uno per volta dopo aver verificato la discesa della kaliemia;

7) la posologia dello spironolattone non dovrebbe eccedere $i$ 25-50 mg/die quando il farmaco è impiegato in combinazione con ACE-inibitori o sartani in pazienti con multipli fattori di rischio per RTA di tipo IV (rene senile, diabete, scompenso cardiaco con elevato fabbisogno di terapia diuretica, insufficienza renale cronica) e questa associazione sarebbe da evitare del tutto in presenza di eGFR inferiore a $30 \mathrm{~mL} /$ $\min (11)$. Nel paziente privo di questi fattori di rischio o con un singolo fattore predisponente in buon compenso (p. es., diabete ben controllato), lo spironolattone può essere impiegato a dosaggi superiori in una terapia di associazione purché esista un chiaro razionale al suo impiego e venga avviato un programma di monitoraggio biochimico periodico (23);

8) il paziente dovrebbe essere informato circa l'opportunità di sospendere la terapia con farmaci bloccanti il RAS in caso di eventi disidratanti e interventi chirurgici maggiori, consultando il Curante.

\section{Conclusioni}

La RTA di tipo IV è una patologia emergente soprattutto a causa della diffusione di farmaci bloccanti il RAS in pazienti anziani affetti da diabete mellito, scompenso cardiaco e insufficienza renale cronica. L'insorgenza di un ipoaldosteronismo, assoluto o funzionale, e l'iperkaliemia che ne consegue inibiscono la capacità del dotto collettore di eliminare sia $\mathrm{K}^{+}$che $\mathrm{H}^{+}$e inibiscono l'ammioniogenesi. Il quadro clinico è caratterizzato da un'acidosi tubulare distale con AG urinario positivo e da un'iperkaliemia sproporzionata alla funzione renale, in genere moderatamente contratta. L'impiego di alcune misure preventive e una maggiore consapevolezza nell'impiego di farmaci bloccanti il RAS sono essenziali per minimizzare il rischio di questa frequente e pericolosa complicanza.

\section{Riassunto}

L'acidosi tubulare tipo IV (RTA di tipo IV) è una forma di acidosi metabolica ipercloremica causata da un ipoaldosteronismo assoluto o funzionale, che determina un deficit dell'acidificazione distale attraverso un'inibizione dell'ammoniogenesi e dell'escrezione di $\mathrm{H}^{+}$a livello del dotto collettore. L'eziologia è spesso multifattoriale e include disordini che determinano una riduzione dei livelli di aldosterone o della sensibilità del dotto collettore alle azioni dell'ormone. I farmaci che inibiscono il sistema renina-angiotensina (RAS) a qualunque livello aumentano il rischio di sviluppare una RTA di tipo IV, soprattutto quando sono impiegati in associazione in pazienti anziani affetti da diabete mellito, scompenso cardiaco e insufficienza renale cronica oppure portatori di trapianto renale. La diagnosi si basa sul riscontro di un'iperkaliemia sproporzionata alla funzione renale associata a un'acidosi metabolica con anion gap sierico normale e anion gap urinario positivo; il pH urinario può essere inferiore a 5.5. Vari elementi laboratoristici consentono la diagnosi differenziale con l'acidosi tubulare distale di tipo 1. L'impatto clinico della RTA di tipo IV sta diventando sempre più rilevante a causa della diffusione di farmaci bloccanti il RAS in pazienti anziani già affetti da condizioni a rischio per questa complicanza, contribuendo a una rivalutazione critica delle indicazioni ad alcune terapie di associazione. Lo sviluppo di iperkaliemia pone in questi contesti un dilemma terapeutico, in quanto proprio i pazienti a maggior rischio per RTA di tipo IV sono quelli che possono trarre il massimo vantaggio cardiovascolare dal blocco farmacologico del RAS. È, quindi, essenziale trovare un punto di equilibrio nel rapporto rischio/beneficio nel singolo paziente, prevenendo la RTA di tipo IV con opportune misure.

Parole chiave: Acidosi tubulare, Iperkaliemia, Sistema renina-angiotensina, Aldosterone, Diabete, Insufficienza renale cronica

Dichiarazione di conflitto di interessi: Gli Autori dichiarano di non avere conflitto di interessi.

Contributi economici agli Autori: Gli Autori dichiarano di non aver ricevuto sponsorizzazioni economiche per la preparazione dell'articolo. 


\author{
Indirizzo degli Autori: \\ Dr. Marco Quaglia \\ Nefrologia e Trapianto \\ Dipartimento di Medicina Traslazionale \& \\ Interdisciplinary Research Center \\ Autoimmune Disease (IRCAD) \\ "Amedeo Avogadro" University \\ Ospedale "Maggiore Della Carità" \\ Corso Mazzini 18 \\ 28100 Novara \\ marco.quaglia@gmail.com
}

\section{Bibliografia}

1. Both T, Zietse R, Hoorn EJ, et al. Everything you need to know about distal renal tubular acidosis in autoimmune disease. Rheumatol Int. 2014; 34 (8): 1037-45.

2. Rothstein M, Obialo C, Hruska KA. Renal tubular acidosis. Endocrinol Metab Clin North Am. 1990; 19 (4): 869-87.

3. Emmet M, Sterns R. Pathophysiology of renal tubular acidosis and the effect on potassium balance. Disponibile su: http:// www.uptodate.com/contents 2014.

4. William F, Young J. Etiology, diagnosis and treatment of hypoaldosteronism (type 4 RTA). Disponibile su: http:/www.uptodate.com/contents 2014.

5. Burton D Rose, Theodore W Post. Chapter 11: Renal hydrogen excretion. In: Clinical Physiology of acid-base and electrolyte disorders. 2005; 328-47 Ed. McGraw-Hill (5 ed.).

6. Burton D Rose, Theodore W Post. Chapter 12: Potassium Homeostasis. In: Clinical Physiology of acid-base and electrolyte disorders. 2005; 372-97. Ed. McGraw-Hill (5 ed.).

7. Karet FE. Mechanisms in hyperkalemic tubular acidosis. J Am Soc Nephrol. 2009; 20: 251-4.

8. Kurtzmann NA. Disorders of distal acidification. Kidney Int. 1990; 38: 720-7.

9. Matsuda O, Nonoguchi H, Tomita K, et al. Primary role of hyperkalemia in the acidosis of hyporeninemichypoaldosteronism. Nephron. 1988; 49: 203-9.

10. Palmer BF. Regulation of Potassium Homeostasis. Clin J Am Soc Nephrol. 2014.

11. Palmer BF. Managing hyperkalemia caused by inhibitors of the rennin-angiotensin-aldosterone system. N Engl J Med. 2004; 351 (6): 585-92.

12. De Fronzo RA. Hyperkalemia and hyporeninemic hypoaldosteronism. Kidney Int. 1980; 17: 118-34.

13. Perazella MA. Drug-induced hyperkalemia: old culprits and new offenders. Am J Med. 2000; 109: 307-14.

14. Arruda J, Battle D, Sehy J, Roseman M, Baronowsy R, Kurtzman NA. Hyperkalemia and renal insufficiency: role of selective aldosterone deficiency and tubular unresponsiveness to aldosterone. Am J Nephrol. 1981; 1: 160-7.

15. Yoon HE, Choi BS. The renin-angiotensin system and aging in the kidney. Korean J Intern Med. 2014; 29 (3): 291-5.

16. Palmer BF, Levi M. Effect of aging on renal function and disease. In: Brenner BM, ed. Brenner and Rector's the kidney, $5^{\text {th }}$ ed. Vol 2 Philadelphia: WB Saunders. 1996; 2274-96.
17. Perazella MA, Mahnensmith RL. Hyperkalemia in the elderly: drugs exacerbate impaired potassium homeostasis. J Gen Intern Med. 1997; 12 (10): 646-56.

18. Battle DC, Arruda JA, Kurtzman NA. Hyperkalemic distal renal tubular acidosis associated with obstructive uropathy. N Engl J Med. 1981; 304: 373-80.

19. Sabatini S, Kurtzman NA. Enzyme activity in obstructive uropathy: basis for salt wastage and the acidification defect. Kidney Int. 1990; 37: 79-84.

20. Petrasek D, Jensen G, Tuck M, Stern N. In vitro effects of insulin on aldosterone production in rat zona glomerulosa cells. Life Sci. 1992; 50 (23): 1781-7.

21. Robles NR, Cerezo I, Hernandez-Gallego R. Renin-angiotensin system blocking drugs. J Cardiovasc Pharmacol Ther. 2014; 19 (1): 14-33.

22. Volpe M, Danser AH, Menard J, et al. Inhibition of the reninangiotensin-aldosterone system: is there room for dual blockade in the cardiorenal continuum? J Hypertens. 2012; 30 (4): 647-54.

23. Palmer BF. Renal dysfunction complicating the treatment of hypertension. N Eng J Med. 2002; 347: 1256-61.

24. Malik SI, Naqvi R, Ahmed E, Zafar MN. Prevalence and risk factors of renal tubular acidosis after kidney transplantation. J Pak Med Assoc. 2011; 61 (1): 23-7.

25. Keven K, Ozturk R, Sengul S, et al. Renal tubular acidosis after kidney transplantation-incidence, risk factors and clinical implications. Nephrol Dial Transplant. 2007; 22 (3): 906-10.

26. Watanabe S, Tsuruoka S, Vijayakumar S et al. Cyclosporin A produces distal renal tubular acidosis by blocking peptidyl prolyl cis-trans isomerase activity of cyclophilin. Am J Physiol Renal Physiol. 2005; 288 (1): F40-7.

27. Schwarz C, Benesch T, Kodras K, Oberbauer R, Haas M. Complete renal tubular acidosis late after kidney transplantation. Nephrol Dial Transplant. 2006; 21 (9): 2615-20.

28. Weir MR, Rolfe M. Potassium homeostasis and renin-angiotensin-aldosterone system inhibitors. Clin J Am Soc Nephrol. 2010; 5 (3): 531-48.

29. St Peter WL, Odum LE, Whaley-Connell AT. To RAS or not to RAS? The evidence for and cautions with renin-angiotensin system inhibition in patients with diabetic kidney disease. Pharmacotherapy. 2013; 33 (5): 496-514.

30. Werner C, Pöss J, Böhm M. Optimal antagonism of the ReninAngiotensin-aldosterone system: do we need dual or triple therapy? Drugs. 2010; 70 (10): 1215-30.

31. Kuijvenhoven MA, Haak EA, Gombert-Handoko KB, Crul M. Evaluation of the concurrent use of potassium-influencing drugs as risk factors for the development of hyperkalemia. Int J Clin Pharm. 2013; 35 (6): 1099-104.

32. Oster JR, Singer I, Fishman LM. Heparin-induced aldosterone suppression and hyperkalemia. Am J Med. 1995; 98 (6): 575-86.

33. Khosla N, Kalaitzidis R, Bakris GL. Predictors of hyperkalemia risk following hypertension control with aldosterone blockade. Am J Nephrol. 2009; 30 (5): 418-24.

34. Juurlink DN, Mamdani MM, Lee DS, et al. Rates of hyperkalemia after publication of the Randomized Aldactone Evaluation Study. N Engl J Med. 2004; 351 (6): 543-51.

35. Dafnis E, Kurtzman NA, Sabatini S. Effect of lithium and amiloride on collecting tubule transport enzymes. J Pharmacol Exp Ther. 1992; 261 (2): 701-6.

36. Kleyman TR, Roberts C, Ling BN. A mechanism for pentami- 
dine-induced hyperkalemia: inhibition of distal nephron sodium transport. Ann Intern Med. 1995; 122 (2): 103-6.

37. Lam N, Weir MA, Juurlink DN, et al. Hospital admissions for hyperkalemia with trimethoprim-sulfamethoxazole: a cohort study using health care database codes for 393,039 older women with urinary tract infections. Am J Kidney Dis. 2011; 57 (3): 521-3.

38. Rodríguez Soriano J. Renal tubular acidosis: the clinical entity. J Am Soc Nephrol. 2002; 13 (8): 2160-70.

39. Batlle DC, Hizon M, Cohen E, Gutterman C, Gupta R. The use of the urinary anion gap in the diagnosis of hyperchloremic metabolic acidosis. N Engl J Med. 1988; 318 (10): 594-9.

40. Walsh SB, Shirley DG, Wrong OM, Unwin RJ. Urinary acidification assessed by simultaneous furosemide and fludrocortisone treatment: an alternative to ammonium chloride. Kidney Int. 2007; 71 (12): 1310-6.
41. Caramelo C, Bello E, Ruiz E, et al. Hyperkalemia in patients infected with the human immunodeficiency virus: involvement of a systemic mechanism. Kidney Int. 1999; 56 (1): 198-205.

42. Golembiewska E, Ciechanowski K. Renal tubular acidosis-underrated problem? Acta Biochim Pol. 2012; 59 (2): 213-7.

43. Sen S, Sabırlı S, Ozyiğit T, Uresin Y. Aliskiren: review of efficacy and safety data with focus on past and recent clinical trials. Ther Adv Chronic Dis 2013; 4 (5): 232-41.

44. FDA Drug Safety Communication: New Warning and Contraindication for blood pressure medicines containing aliskiren. Disponibile su: www.fda.gov/drugs/drugsafety/ucm300889.htm Feb 2013.

45. Koppe L, Klich A, Dubourg L, Ecochard R, Hadj-Aissa A. Performance of creatinine-based equations compared in older patients. J Nephrol. 2013; 26 (4): 716-23. 